\title{
Potential Predictive Factors for Clinical Outcomes Following Progressive Pain Reduction Associated With Conservative Treatment for Recently Diagnosed Osteoporotic Vertebral Fractures: A Case Control Study
}

Yosuke Shibao ( $\nabla$ shiba_you_0720@yahoo.co.jp )

Department of Orthopedic Surgery, Faculty of Medicine, University of Tsukuba

Masao Koda

Department of Orthopedic Surgery, Faculty of Medicine, University of Tsukuba

Yosuke Takeuchi

Department of Orthopedic Surgery and Sports Medicine, University of Tsukuba Hospital Mito Clinical Education and Training Center Mito Kyodo General Hospital

\section{Tomoyuki Asada}

Department of Orthopedic Surgery, Faculty of Medicine, University of Tsukuba

\section{Kosuke Sato}

Department of Orthopedic Surgery, Faculty of Medicine, University of Tsukuba Mamoru Kono

Department of Orthopedic Surgery, Faculty of Medicine, University of Tsukuba Fumihiko Eto

Department of Orthopedic Surgery, Faculty of Medicine, University of Tsukuba

Kousei Miura

Department of Orthopedic Surgery, Faculty of Medicine, University of Tsukuba

Hiroshi Noguchi

Department of Orthopedic Surgery, Faculty of Medicine, University of Tsukuba

Hiroshi Takahashi

Department of Orthopedic Surgery, Faculty of Medicine, University of Tsukuba

\section{Toru Funayama}

Department of Orthopedic Surgery, Faculty of Medicine, University of Tsukuba

\section{Masaki Tatsumura}

Department of Orthopedic Surgery and Sports Medicine, University of Tsukuba Hospital Mito Clinical Education and Training Center Mito Kyodo General Hospital

Masashi Yamazaki

Department of Orthopedic Surgery, Faculty of Medicine, University of Tsukuba 


\section{Research Article}

Keywords: Osteoporotic vertebral fracture, Conservative treatment, Visual analog scale

Posted Date: February 17th, 2022

DOI: https://doi.org/10.21203/rs.3.rs-1316940/v1

License: (c) (i) This work is licensed under a Creative Commons Attribution 4.0 International License. Read Full License 


\section{Abstract}

\section{Introduction}

The purpose of the present study was to elucidate a potential predictive factor for poor clinical outcomes in the conservative treatment of osteoporotic vertebral fractures.

\section{Materials and Methods}

This is a study of the vertebrae of 41 patients selected from 63 patients with recent osteoporotic fractures admitted to our hospital from April 2013 to March 2015. Patients with dementia were excluded. We analyzed visual analog scale (VAS: 0-100 mm) scores and dynamic radiographic images over time for up to 12 weeks after admission. Our statistical analyses were designed to identify factors that were significantly associated with pain prolongation.

\section{Results}

Logistic regression analysis using a stepwise method revealed that the independent factor significantly associated with failure for $50 \%$ pain reduction at the final follow-up visit was VAS reduction rate at week $3(\mathrm{OR}=9.0,95 \% \mathrm{Cl} 0.5-17.6, p=0.04)$.

\section{Conclusions}

Patients who responded well to treatment reported a reduction in pain during the first 3 weeks of treatment versus nonresponders who did not. It may be possible to predict the prognosis of osteoporotic vertebral fractures early during the first 3 weeks after hospital admission.

\section{Introduction}

In principle, osteoporotic vertebral fractures (OVF) can be treated conservatively and the clinical outcomes of treatment with rest and orthoses have been well reported [1,2] .However, there is no standardized treatment regimen and the outcome of OVF may be affected by multiple factors, such as age, sex, underlying diseases, and the type of fracture, all of which are reportedly predictive of prognosis [3]. We prioritize the process of pain reduction when assessing patients' conditions; it is among the most important objectives of treatment considering that when pain is prolonged, an underlying cause generally exists. Most patients who develop issues, such as delayed palsy, complain of severe, long-term pain. Thus, a focus on the process of pain reduction may inform clinical prognosis predictions. A first step to verify this hypothesis is to identify how pain changes in patients who respond well to conservative treatment. The aim of the present study was to elucidate whether the an early reduction in pain can be one of the predictive factors for clinical outcomes associated with the conservative treatment for OVF.

\section{Methods}


The study subjects were 41 patients ( 5 men and 36 women) selected from 63 patients with recently diagnosed OVFs who were admitted to our hospital from April 2013 to March 2015(Fig. 1). Patients with dementia and those for whom clinical and imaging assessments for 12 weeks after admission were not available were excluded. Pain was assessed using a visual analog scale (VAS; 0-100 mm) score at admission and after 3,6 , and 12 weeks. The degree of vertebral instability was confirmed using dynamic radiographs of patients in standing and supine positions that are in accordance with the method of Abe et al [4]. Gross instability was defined as a difference of $\geq 5$ degrees in the vertebral wedge angle on a lateral radiograph with vertebral sway or the presence of a vacuum cleft. Kyphotic angle was defined as an angle formed by the cranial end plate of the vertebral body just above the fractured vertebra and the caudal end plate of the vertebra just below the fractured vertebral body.

A favorable clinical outcome was defined as a $50 \%$ or greater reduction in pain at the final 12-month visit. Failure was defined as cases with no reduction in pain, or when surgical intervention was required. We performed statistical analyses using a $50 \%$ or greater reduction in pain at the final visit as an objective variable and course of pain reduction (VAS at admission and VAS reduction rate at week 3 , as well as the following imaging factors: vertebral instability at admission and vertebral instability, kyphotic angle, and wedge angle-all at week 3 ) as explanatory variables. Factors identified with a significance of $p<0.1$ in the initial univariate analysis were used in further analyses. Possible confounding among explanatory factors during univariate analysis was assessed. Logistic regression analysis using a stepwise method was performed to identify possible independent risk factors which had a significant impact on clinical outcomes. Data are expressed as mean \pm standard deviation (SD). The odds ratio (OR) for the incidence of failure and $95 \%$ confidence intervals $(\mathrm{Cl})$ were calculated as an approximation of the relative risk estimates. All statistical analyses were conducted using JMP 15 (SAS Institute Inc., Cary, NC, USA).

\section{Results}

The demographic characteristics of patients are shown in Table 1. The mean follow-up was 7.6 months. The mean VAS score improved over time from $67 \mathrm{~mm}$ at admission to $31 \mathrm{~mm}$ at week 3, $24 \mathrm{~mm}$ at week 6 , and $20 \mathrm{~mm}$ at week 12. The mean vertebral instability also improved from 4.39 degrees at week 3, 2.86 degrees at week 6 , and 2.21 degrees at week 12. Four of the 41 patients required surgery (percutaneous vertebroplasty in three cases and a posterior spinal fusion in one case) owing to severe pain which was classified as a reduction in pain of less than $50 \%$ since admission. In addition, there were four cases with no improvement in pain (Fig. 2). Using a univariate analysis, the VAS reduction rate at week 3 , vertebral instability at admission, and vertebral instability at week 3 were identified as potencial significant factors $(p<0.1)$ for predicting failure and were used in subsequent analyses. Logistic regression analysis revealed that the independent factor significantly associated with failure to achieve $\geq 50 \%$ reduction in pain by the final follow-up visit was a VAS reduction rate at week $3(\mathrm{OR}=9.0,95 \% \mathrm{Cl} 0.5-17.6, p=0.04)$ (Table 2). The receiver-operator characteristic analysis revealed that the cutoff value for the VAS reduction rate at week 3 was $27 \%$ or grater (Fig. 3), indicating that patients achieving a $27 \%$ reduction in pain at week 3 had a $50 \%$ or grater reduction in pain by the final follow-up visit. 
Table 1

Baseline characteristics

\begin{tabular}{|lll|}
\hline & \multicolumn{2}{l|}{ Clinical Outcome* } \\
\hline Characteristic & Favorable & Unfavorable \\
\hline Patients, $\mathrm{n}(\%)$ & $29(78.3)$ & $8(21.7)$ \\
\hline Age, years & $80.2 \pm 9.6$ & $84.5 \pm 2.5$ \\
\hline Female gender, $\mathrm{n}(\%)$ & $26(89.7)$ & $6(75.0)$ \\
\hline BMI, kg/m ${ }^{2}$ & $23.5 \pm 3.7$ & $21.9 \pm 4.8$ \\
\hline BMD, g/cm ${ }^{2}$ & $0.8 \pm 0.27$ & $1.10 \pm 0.19$ \\
\hline Medication use for osteoporosis preinjury & $3(10.3)$ & $2(6.9)$ \\
\hline Use of internal oral painkillers, $\mathrm{n}(\%)$ & $16(62.5)$ & $3(37.5)$ \\
\hline NSAIDs & $3(10.3)$ & $1(12.5)$ \\
\hline Acetaminophen & & \\
\hline${ }^{*}$ Clinical outcome was for a reduction in pain of $\geq 50 \%$ by 12 -week. & \\
\hline BMD, bone mineral density; BMl, body mass index; NSAIDs, nonsteroidal antiinflammatory drugs \\
\hline
\end{tabular}


Table 2

Risk factors associated with the failure of conservative treatment to reduce the pain of osteoporotic vertebral fractures by $\geq 50 \%$

\begin{tabular}{|ll|}
\hline Type of statistical analysis & $p$-value \\
\hline Univariate analysis* & \\
\hline VAS & 0.95 \\
\hline At admission & $0.07^{*}$ \\
\hline Reduction rate at 3 weeks & \\
\hline At admission & $0.03^{*}$ \\
\hline Reduction rate at 3 weeks & $0.05^{\star}$ \\
\hline Change in vertebral angle at 3 weeks & 0.5 \\
\hline Kyphotic & 1.0 \\
\hline Wedge & \\
\hline Stepwise logistic regression** & $0.04^{\star *}$ \\
\hline VAS reduction rate at 3 weeks & 0.3 \\
\hline Instability at admission & 0.3 \\
\hline Instability at 3 weeks & \\
\hline Levels of significance per analysis were: ${ }^{*} p<0.1$ and ${ }^{* *} p<0.05$ \\
\hline
\end{tabular}

\section{Discussion}

In the present case series, we determined that a poor pain reduction rate $(\sim 27 \%) 3$ weeks after the diagnosis of an OVF that caused pain was a factor significantly associated with inadequate pain relief at final follow-up (14 months). Until now, few studies have addressed the process of pain recovery in patients with OVF treated with conservative therapy. Based on the experience of individual doctors in clinical practice or at hospitals managing these patients, wide variations in the initial treatments for OVF -such as drug administration, bracing, bedrest for a set duration, admission to a hospital, and so forthare among the possible reasons for a lack of reports addressing the course of pain reduction in the past. This wide variation makes it challenging to compare the actual time course of pain reduction between patients who received different therapeutic regimens. In contrast, in this case series we applied a conservative standardized treatment regimen that included 2 weeks of bedrest followed by rehabilitative treatment that required a rigid Jewett spinal brace to be worn; this enabled us to analyze the course of pain reduction over time. We consider this one of the strong points of our present study. 
Toyoda and colleagues performed a stepwise cluster analysis using VAS scores and identified four different patterns of lumbar pain progression after OVF [5]. In that report, cluster 4 included the patients who initially exhibited moderate to strong pain without pain reduction over the clinical course. Another cluster included patients with initial moderate pain that was rapidly attenuated. In the present study, patients in the group who experienced an unfavorable level of pain reduction ( $<50 \%$ at 3 weeks similarly exhibited an inadequate reduction in their pain level 12 weeks after hospital admission for OVF. Results at the final follow-up visit largely overlap with the cluster 4 results in the report of Toyoda et al. Both reports showed that a failure to adequately relieve pain in the relatively early phase of treatment is significantly associated with inadequate pain relief at final follow-up. In other words, the pain reduction rate at a relatively early phase of OVF could be one of the possible predictive factors for the prognosis of pain reduction. Further exploration with a larger prospective cohort in the near future is prerequisite to being able to elucidate the true usefulness of using pain mitigation early in the course of treatment as a predictive factor for pain prognosis in patients with OVF.

Previously, Toyone et al. reported that vertebral instability in the early phase after injury was significantly associated with pain reduction at final follow-up [6]. In the present study, univariate analysis revealed that vertebral instability 3 weeks after diagnosis of OVF was significantly associated with final outcomes; this aligns with the report of Toyone and colleagues. A possible reason for a discrepancy between the two studies is the cutoff of the dichotomous objective variable, $\geq 50 \%$ pain reduction. Therefore, it might be considered premature to conclude that vertebral instability is unassociated with final outcome.

The present study has several strengths and limitations. We consider that the ease of evaluation is a strength of our study in addition to only evaluating pain intensity after an appropriate period of conservative therapy. Furthermore, the conservative standardized treatment regimen lends strength to our results. In contrast, the latter approach could hinder the generalizability of our results to patients receiving other types of conservative treatment. The limitations of this study included the small number of patients, a short follow-up period, and the fact that we did not consider other factors associated with the clinical course, such as fracture type and underlying disorders. The retrospective nature of the present study is another possible limitation given that we could not consider the patients who discontinued the study.

Currently, we apply conservative standardized therapy regimens to almost all patients with OVF as a way of screening. If the reduction in pain achieved via initial conservative treatment is inadequate, we consider other treatment options that include surgery. Our study results are encouraging and support our current treatment strategy.

\section{Conclusion}

Pain reduction rate 3 weeks after initiating conservative treatment for a newly diagnosed OVF is among the potential predictive factors for a prognosis of inadequate pain reduction at final follow-up.

- I confirm that I understand BMC Musculoskeletal Disorders is an open access journal that levies an article processing charge per articles accepted for publication. By submitting my article I agree to pay 
this charge in full if my article is accepted for publication.

- No, I declare that the authors have no competing interests as defined by BMC, or other interests that might be perceived to influence the results and/or discussion reported in this paper.

- The results/data/figures in this manuscript have not been published elsewhere, nor are they under consideration (from you or one of your Contributing Authors) by another publisher.

- I have read the BMC journal policies on author responsibilities and submit this manuscript in accordance with those policies.

- All of the material is owned by the authors and/or no permissions are required.

- I am the author responsible for the submission of this article and I accept the conditions of submission and the BMC Copyright and License Agreement as detailed above.

\section{Abbreviations}

VAS

visual analog scale OVF:Osteoporotic vertebral fracture SD:standard deviation OR:odds ratio Cl:confidence intervals

\section{Declarations}

\section{Ethics approval and consent to participate}

This study was approved by the ethical committee of Tsukuba Clinical Research and Development Organization (H30-087). Verbal informed consent was obtained from all participants. And, we confirm that all methods were performed in accordance with the relevant guidelines.

\section{Consent for publication}

Not applicable.

\section{Availability of data and materials}

All data generated or analyzed during this study are included in this published article [and its supplementary information files].

\section{Competing interests}

The authors declare that they have no competing interests.

\section{Funding}

Not applicable.

\section{Authors' contributions}


YS and YT designed the study. YS wrote the article. TA, KS, MK, FE, KM, HN, HT, TF, MT and MY reviewed the article. MK collected and analyzed the data. All authors read and approved the final manuscript.

\section{Acknowledgements}

Not applicable.

\section{Authors' information}

${ }^{1}$ Department of Orthopeadic Surgery, Faculty of Medicine, University of Tsukuba, Ibaraki, 1-1-1 Tennodai, Tsukuba, Ibaraki 305-8575, Japan

2 Department of Orthopedic Surgery, Tokyo Medical University Ibaraki Medical Center, Ami, Ibaraki, Japan

\section{References}

1. Kim DY, Lee SH, Jang JS, Sang CK, Lee HY(2004) Intravertebral vacuum phenomenon in osteoporotic compression fracture report of 67 cases with quantitative evaluation of intravertebral instability. J Neurosurg Spine 100:24-31. doi: 10.3171/spi.2004.100.1.0024.

2. Wu CT, Lee SC, Lee ST, Chen JF(2006) Classification of symptomatic osteoporotic compression fractures of the thoracic and lumbar spine. J Clin Neurosci 13:31-38. doi:

10.1016/j.jocn.2004.11.021.

3. Tsujio T, Nakamura $H$, Terai $H$, Hoshino M, Namikawa T, Matsumura A, Kato M, Suzuki A, Takayama K, Fukushima W, Kondo K, Hirota Y, Takaoka K(2011) Characteristic radiographic or magnetic resonance images of fresh osteoporotic vertebral fractures predicting potential risk for nonunion: a prospective multicenter study. Spine 3:1229-1235. doi: 10.1097/BRS.0b013e3181f29e8d.

4. Abe T, Shibao Y, Takeuchi Y, Mataki Y, Amano K, Hiroki S, Miura K, Noguchi H, Funayama T, Koda M,Yamazaki M(2018) Initial hospitalization with rigorous bedrest followed by bracing and rehabilitation as an option of conservative treatment of osteoporotic vertebral fractures in elderly patients: a pilot one arm safety and feasibility study. Arch Osteoporos 13:134. doi: 10.1007/s11657018-0547-0

5. Toyoda H, Takahashi S, Hoshino Masatoshi, Takayama K, Iseki K, Sasaoka R, Tsujio T, Yasuda H, Sasaki T, Kanematsu F, Kono H, Nakamura H(2017) Characterizing the course of back pain after osteoporotic vertebral fracture: a hierarchical cluster analysis of a prospective cohort study. Arch Osteoporos 12:82. doi: 10.1007/s11657-017-0377-5

6. Toyone T, Tadashi Tanaka, Yuichi Wada, Kamikawa K, Ito M, Kimura K, Yamasita T, Matsushita S, Shiboi R, Kato D, Kaneyama R, Otsuka M(2006) Changes in vertebral wedging rate between supine and standing position and its association with back pain: a prospective study in patients with osteoporotic vertebral compression fractures. Spine (Phila Pa 1976) 31:2963-2966. doi: 10.1097/01.brs.0000247802.91724.7e. 
Figures

Fig. 1

63 patients with osteoporotic vertebral fractures were screened

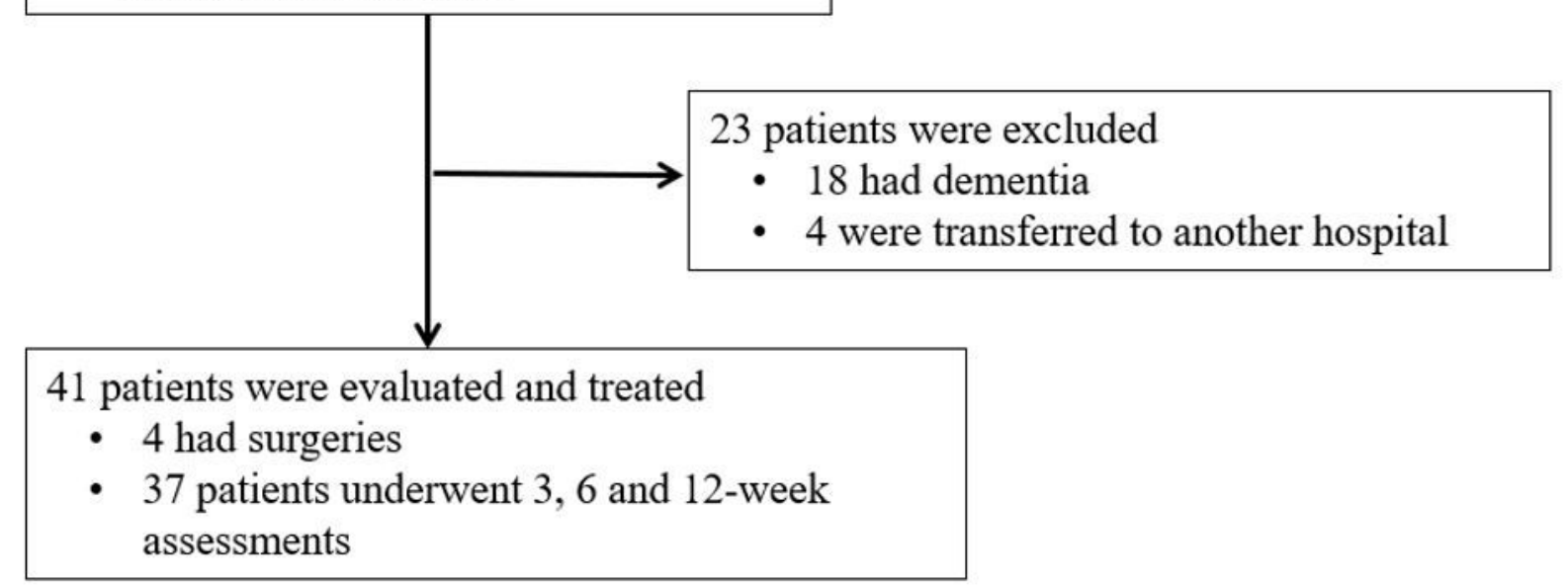

Figure 1

Flow diagram for patient enrollment. 
Fig. 2

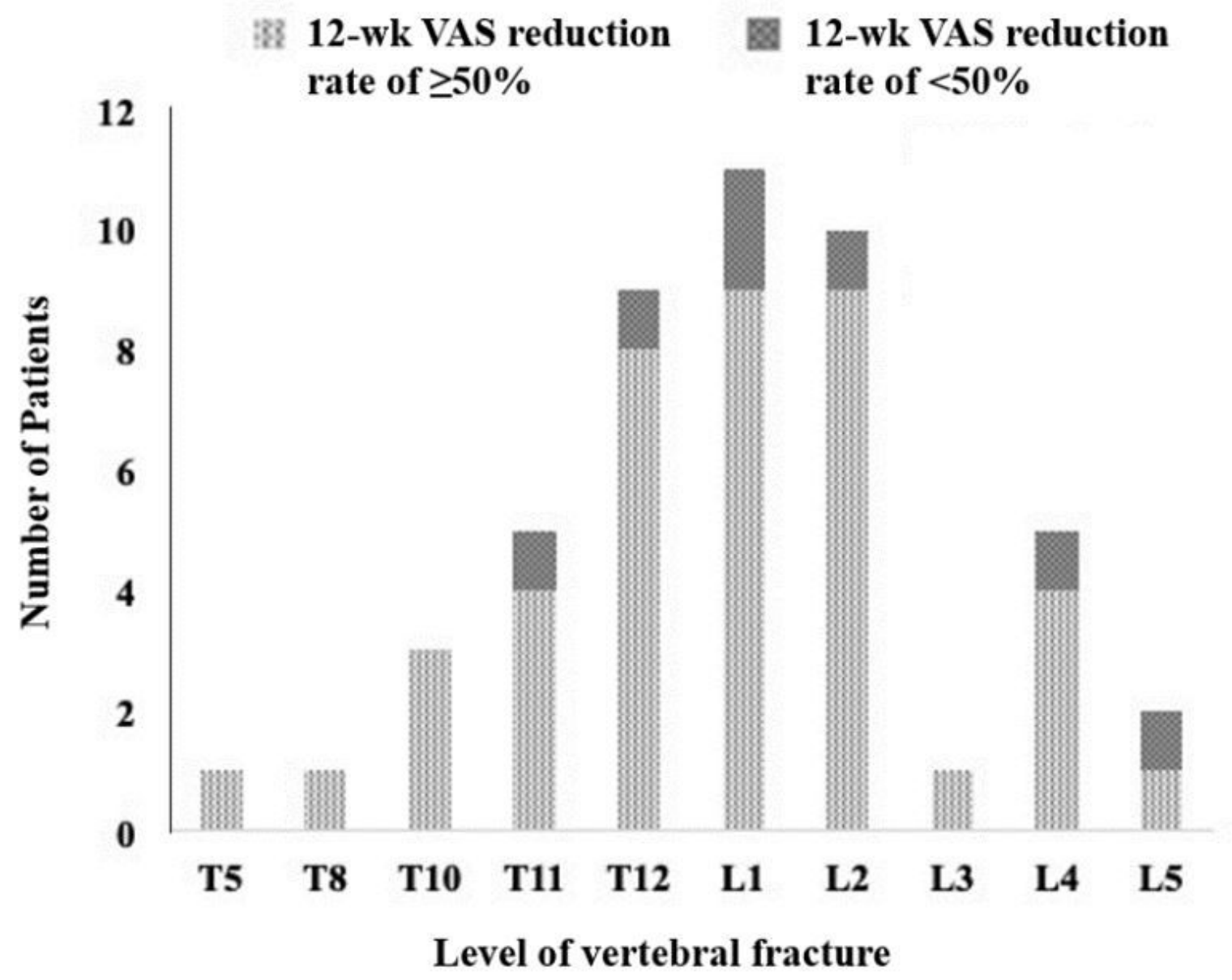

Figure 2

Clinical outcomes at the 12-week assessment by fractured vertebral levels $(n=37)$.

L, lumbar; T, thoracic; VAS, visual analog scale 
Fig. 3

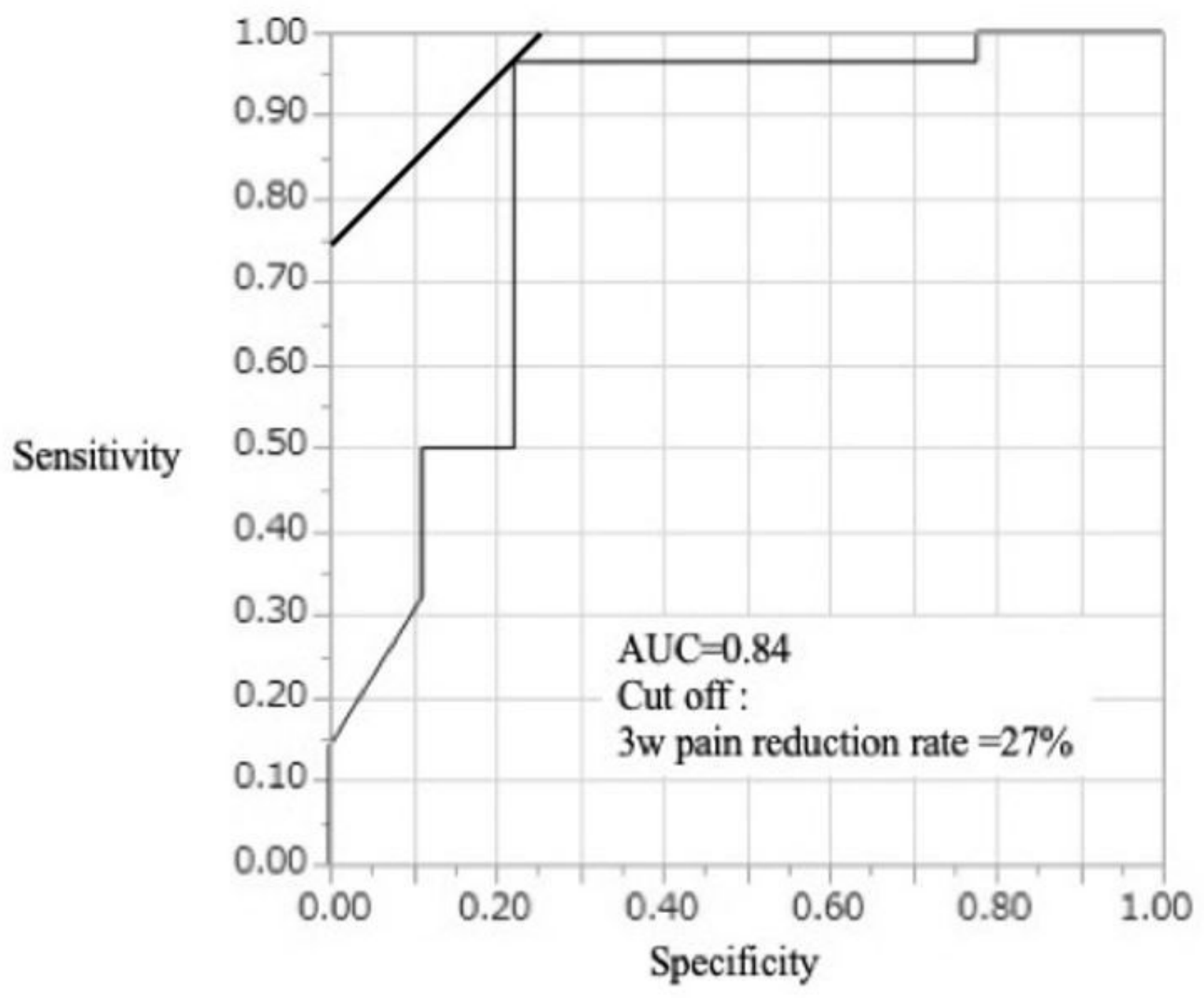

Figure 3

Receiver operating characteristic curve depicting the 3-week pain reduction rate cutoff as $27 \%$. AUC, area under the curve 